\title{
Osteoma gigante en mandíbula. Reporte de Caso.
}

\section{Giant osteoma in jaw. Case Report.}

Carlos Licéaga-Escalera ${ }^{1, a}$, Jorge Del Bosque-Méndez ${ }^{1, b}$, Beatriz Aldape-Barrios ${ }^{2, c}$, Luis Montoya-Pérez ${ }^{1, d}$, Ethien Morelos-Patiño ${ }^{2, e}$, Victor González-Olmedo ${ }^{2, e}$.

\section{RESUMEN}

El osteoma es una neoplasia benigna poco común, que se caracteriza por presentar un crecimiento óseo progresivo, el cual está compuesto por la proliferación bien diferenciada de hueso compacto y/o esponjoso. En el área maxilofacial se presenta con mayor frecuencia en la cara lingual posterior de la mandíbula. Se caracteriza por crecimiento lento y asintomático. El caso describe una paciente de 45 años con un osteoma gigante mandibular de 4 años de evolución tratado quirúrgicamente.

\section{Palabras clave: OSTEOMA, PATOLOGÍA, TERAPIA, MANDÍBULA, TRATAMIENTO, QUIRÚRGICO.}

\begin{abstract}
Osteomas are considered a rare benign neoplasm, characterized by a progressive bone growth, which is composed of a proliferation well differentiated of compact or cancellous bone. In the maxillofacial area when present, commonly found in the posterior lingual surface of the mandible. It is characterized by slow growth and asymptomatic. The case describes a mandibular giant osteoma of 4 year of evolution treated with surgical treatment.
\end{abstract}

Key Words: OSTEOMA, PATHOLOGY, MANDIBLE, SURGERY, THERAPY.

\footnotetext{
1 Hospital Juárez de México. México D. F., México.

2 Facultad de Odontología, Universidad Nacional Autónoma de México. México D. F., México.

a Jefe de Servicio de Cirugía Maxilofacial.

b Jefe de Oncología de Cabeza y Cuello.

- Profesora de Patología Bucal.

d Médico Adscrito de Cirugía Maxilofacial.

e Residente de Cirugía Maxilofacial,
} 


\section{INTRODUCCIÓN}

El osteoma es una lesión benigna compuesta de hueso maduro lamelar. Descrito como una entidad por Jaffe en 1935. Se caracteriza por ser una lesión bien circunscrita, redonda y de crecimiento lento (1). Generalmente se identifica por hallazgos radiográficos (2), es asintomático, cuando se presenta sintomatología se le atribuye a naturaleza compresiva y no invasiva o destructiva.

La etiología no está clara, se han propuesto varias hipótesis como: una anomalía congénita $(1,3)$ Fettissof en 1938 mencionó que se origina a partir de restos embriológicos de perióstio (4), así como Varboncoeur en 1990 agregó la persistencia de cartílago, el cual inicia la formación de hueso $(3,4)$. También se ha propuesto inflamación crónica causando una proliferación neoplásica. Schneider (1980) y Kaplan (1994) sugirieron que el trauma $(5,6)$ y la tracción de la musculatura sobre el perióstio puede favorecer a su desarrollo $(2,6)$ Existe mayor predilección por el sexo masculino (6) Se puede presentar a cualquier edad, existe mayor incidencia entre la tercera y quinta décadas de vida (7). No se han reportado casos en los que sufra transformación maligna $(2,8)$.

Los osteomas se pueden clasificar en centrales los cuales se originan a partir del endostio, periféricos originados del perióstio y los extra esqueléticos que se desarrollan en estructuras de tejido muscular $(2,3,5,6,8)$. Histológicamente se pueden clasificar en compacto o de "marfil" que se presentan con mayor frecuencia en los huesos planos del cráneo, medular con mayor incidencia en huesos largos y mixtos $(2,5,8)$.

La mayoría de los casos se presentan en la región craneofacial, en los senos paranasales, principalmente el seno frontal, de forma esporádica en la mandíbula y se desarrollan en el cóndilo, cortical lingual del cuerpo y en el reborde basal del ángulo $(6,8)$. En el maxilar son menos comunes presentándose en el seno maxilar y tuberosidad.

La presencia de múltiples osteomas en la región maxilomandibular puede formar parte del síndrome de Gardner (poliposis adenomatosa familiar) (8), la cual es una enfermedad autosómica dominante, causada por la mutación en el cromosoma 5q21-22 (9).
Este tipo de síndrome se caracteriza por la presencia de múltiples osteomas en la región facial, presencia de poliposis en el intestino grueso con alta incidencia de transformación maligna, así como odontomas, fibromatosis de la piel, presencia de múltiples quistes sebáceos y dermoides (9) Se pueden presentar dientes supernumerarios y en mala posición. Por lo que se debe descartar la posibilidad de presentar este síndrome $(6,9)$

El diagnóstico se completa con imágenes radiográficas principalmente los endosteales $(6,8,9)$. La imagenología muestra la presencia de la posición y la naturaleza benigna de la patología, siendo la tomografía axial computarizada la más exacta en su localización y relaciones específicas. La mayoría son unilaterales con forma de hongo o pediculados, observándose como una masa radiopaca $(7,10)$.

Los diagnósticos diferenciales deben incluir la exostosis que se presenta asociada a fenómenos traumáticos o inflamatorios de crecimiento limitado a diferencia del osteoma que muestra un crecimiento independiente y características clínicas de neoplasia benigna $(6,8,10)$, osteomielitis esclerosante focal, osteoblastoma, osteosarcoma, displasia fibrosa, odontoma y la enfermedad de Paget $(3,7,11)$.

El tratamiento es la excisión de la lesión $(10,11)$. La indicación para el retiro de esta se basa en la asimetría que pueda llegar a causar modificando la estética, la sintomatología que pueda ocasionar, obstrucción de la vía aérea, dificultad al deglutir $(8,11)$, incluso cabe la posibilidad de no retirarse ya que la mayoría son asintomáticos, pero existe también el riesgo que debido a que es una lesión de crecimiento progresivo desarrolle tamaños considerablemente grandes y pueda alcanzar áreas como la órbita siendo mas difícil su remoción $(9,11)$, la recurrencia es rara $(2,6,7,1)$.

\section{CASO CLÍNICO}

Paciente femenino de 46 años de edad que presenta aumento de volumen en la región submandibular izquierda de 4 años de evolución, (fig. 1) asintomático, del mismo color de la mucosa adyacente, a la palpación dura y fija. Intraoralmente y sistémicamente sin alteraciones. En la ortopantomografía presenta una imagen radiopaca en la zona basal mandibular izquierda de 5 x $4 \mathrm{~cm}$ aproximadamente, (fig. 2) de 
límites bien definidos. Se solicita estudio de tomografía, en donde se aprecia imagen de la misma densidad del tejido óseo, en reconstrucción 3D se aprecia multilobulado y de superficie lisa. Se decide intervención bajo anestesia general, se realiza abordaje intraoral vestibular, se descubre la lesión y se realiza osteotomía con pieza de baja velocidad concluyendo con cinceles y percutor. Finalmente se remodela con fresón y pieza de bajá velocidad. (fig. 3) Se concluye procedimiento suturando la herida.

Se envía espécimen quirúrgico a estudio histopatológico para su descalcificación, que lleva un tiempo prolongado. En la descripción histológica se reporta hueso lamelar vital maduro sin médula ósea y poca actividad osteoclastica. (Fig. 4). Se toma radiografía de control a la semana de postoperatorio, donde se observa adecuado contorno y simetría mandibular. (Fig 5 y 6).

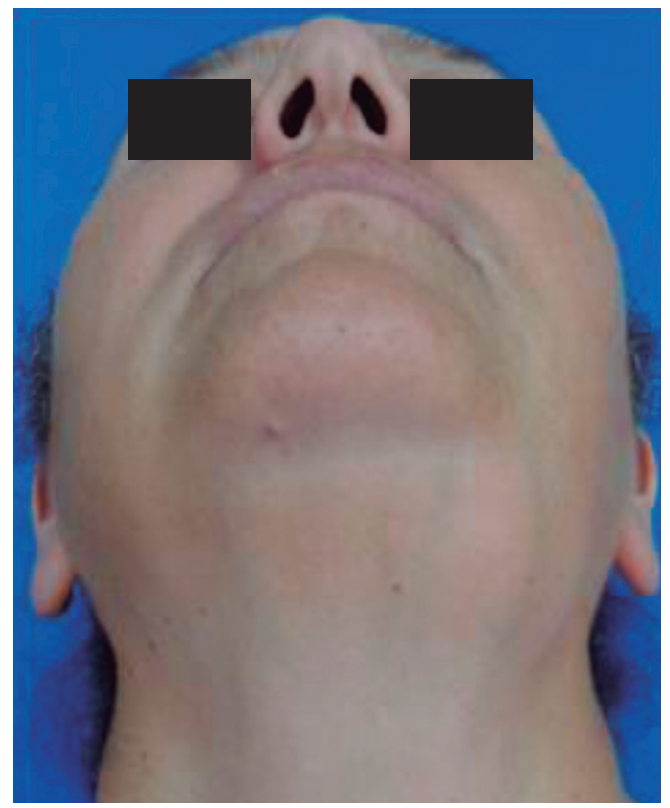

Fig 1. Aumento de volumen submandibular izquierdo.

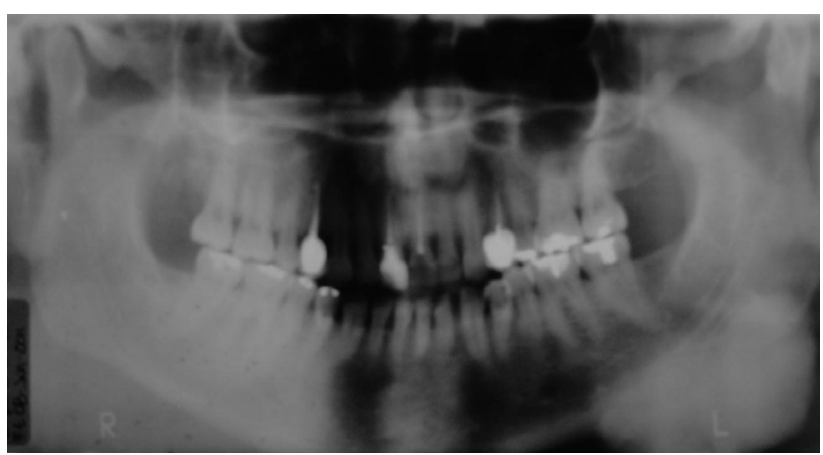

Fig 2. Ortopantomografía pre quirúrgica.

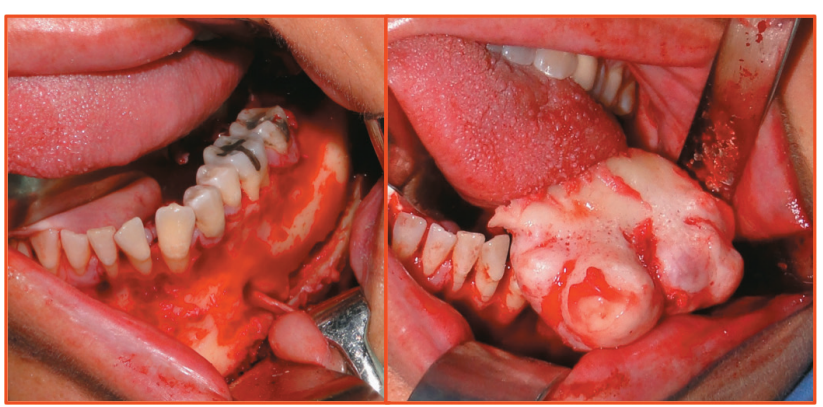

Fig 3. Osteotomía de lesión.

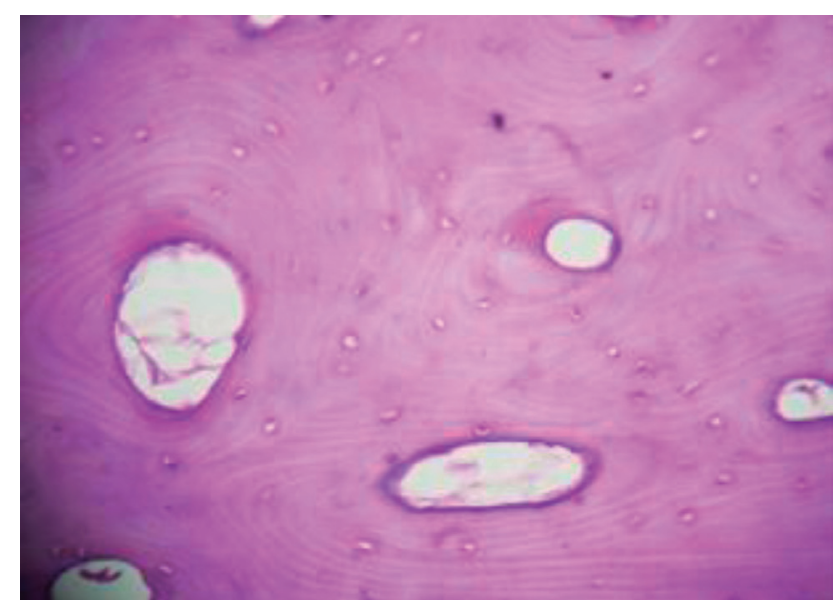

Fig 4. Corte histológico.

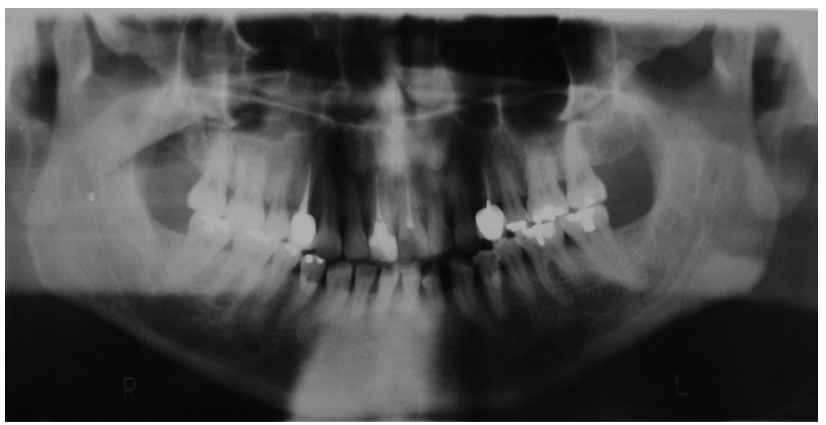

Fig 5. Ortopantomografía de control post quirúrgica.

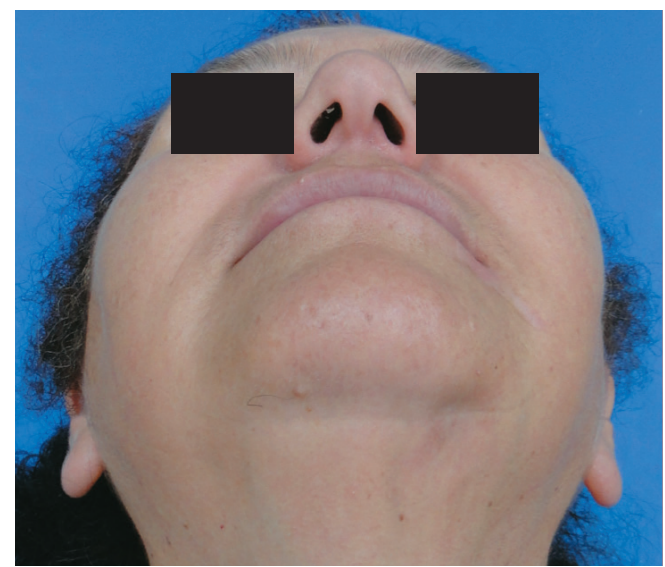

Fig 6. Control postoperatorio. 


\section{DISCUSIÓN}

El osteoma no presenta signos y síntomas, en ocasiones se presenta como una expansión; el hallazgo de este es radiográfico en una visita al odontólogo. Sin etiología definida. Una de la indicaciones para el retiro de esta entidad es cuando se ve comprometida la estética o la función del paciente como la deglución y la dificultad a la respiración. ${ }^{12}$ Siendo el tratamiento la escisión quirúrgica. El diagnóstico es de suma importancia para evitar tratamientos inadecuados, debido a que existen otras entidades patológicas parecidas.

El osteoma es una neoplasia verdadera a diferencia de la exostosis a la que en ocasiones erróneamente se le atribuye este título, en realidad siendo un hamartoma. Otra diferencia es que las exostosis se detienen en su crecimiento en la pubertad, a diferencia del osteoma el cual sigue aumentando su tamaño a través del tiempo $(1,12)$.

En el caso presentado se muestra el gran tamaño que puede alcanzar la lesión, expandiéndose hasta provocar asimetría la cual puede ser fácilmente percibida. El termino de gigante se le atribuye a las lesiones que alcanzan tamaños poco usuales, dentro de la revisión se encontró que este término se le dio a lesiones dentro del rango radiográfico entre $3 \times 3 \mathrm{~cm}$. a $6 \times 4.5 \mathrm{~cm}$. y de $4 \times 3.7 \times 3.5 \mathrm{~cm}$. a $3.3 \times 5.2 \times 8.7 \mathrm{~cm}$. en especímenes enviados al patólogo. Nuestro caso se encuentra situado en el rango, por lo que se le atribuyo el título de gigante $(1,3,8,10,12,13)$.

\section{CONCLUSIÓN}

El osteoma es una entidad patológica que se presenta de manera esporádica en la región maxilofacial, el diagnóstico por lo regular es a través de hallazgo radiográfico, aunque en algunas ocasiones alcanza tamaños considerables que ocasiona deformidades. Es importante la correlación histopatológica e imagenológica para un diagnóstico adecuado, esto para distinguirlo cuando se presente en asociación al síndrome de Gardner. El tratamiento de la lesión es la excisión de la lesión.

\section{CORRESPONDENCIA}

Dr. Victor Manuel González Olmedo

Av. Instituto Politécnico Nacional No.5160
Col. Magdalena de las Salinas Consultorio 27,

Primer Piso, Consulta Externa. C.P. 07760

México, Distrito Federal.

Teléfono: 57477560

Correo electrónico: victor_go7@hotmail.com

\section{REFERENCIAS BIBLIOGRÀFICAS}

1. Swanson KS, Guttu RL, Miller ML. Gigantic Osteoma of the Mandible: Report of a Case. American Association of Oral and Maxillofacial Surgeons.1992;50:635-8.

2. Rugierro RB, Rizzo S, Fiandrino G, Lupi S, Galioto S. Mandibular traumatic peripheral osteoma: a case report. Oral Surgery, Oral Medicine, Oral Pathology, Oral Radiology and Endodontology. 2011;112(6):44-8.

3. Göksel SK, Mehmet MÖ, Sare Şipal S, Ümit E. Gigantic Peripheral Osteoma of the Mandible: A Case Report. Journal of Clinical and Experimental Dentistry. 2010;2: e160-2.

4. Fetisoff AG. Pathogenesis of Osteomas of the nasal accessory sinuses. Annals of Otology, Rhinology, and Laryngology. 1938;404.

5. Colletti G, Autelitano L, Rabbiosi D, Tewfik K, Frigerio A, Biglioli F. Parosteal osteoma arising in an iliac bone graft used for mandibular reconstruction. Journal of Maxillofacial Surgery. 2012;70(8):477-80.

6. Goudar G, Ravi Kumar R, Manjunath GA, Mahadesh J. Osteoma of the Mandible. Journal of Dental Sciences \& Research. 2011;2 (1):116-22.

7. Longo F, Califano L, De Maria G, Ciccarelli R. Solitary osteoma of the mandibular ramus: report of a case. Journal of Maxillofacial Surgery. 2001;59(6):698-700.

8. Kerckhaert A, Wolvius E, Van Der Wal K, Oosterhuis JW. A giant osteoma of the mandible: Case report. Journal of Cranio-Maxillofacial Surgery. 2005;33:282-5.

9. Lagha NB, Galeazzi JM, Chapireau D, Oxeda P, Bouhnik Y, Maman L. Surgical Management of Osteoma Associated With a Familial Gardner's Syndrome. J Oral Maxillofac Surg. 2007;65:1234-40.

10.Donohué-Cornejo, Franco LE, Albarrán-Vergara S, Gaitán LA. A giant osteoma of the mandible: Case report. Journal of Cranio-Maxillofacial Surgery 2005;33(4):282-5.

11.Sayan NB, Ucok C, Karasu HA, Gunhan O. Peripheral Osteoma of the Oral and Maxillofacial Region: A Study of 35 New Cases. American Association of Oral and Maxillofacial Surgeons. 2002;60:1299-301.

12.Seo-Young An, Chang-Hyeon An, Karp-Shik Choi. Giant Osteoma of the Mandible Causing Brething Problem. Korean Journal of Oral and Maxillofacial Radiology 2006;36: 217-20.

13. Gawande P, Deshmukh V, Garde JB. A Giant Osteoma of the Mandible. Journal of Maxillofacial and Oral Surgery 2011 Mar;1-6.

Recibido : 05-06-2013

Aceptado: 30-10-2013 